\title{
QUANTUM TRANSPORT IN THIN-FILM STRUCTURES
}

\author{
B. BULKa \\ Institute of Molecular Physics, Polish Academy of Sciences \\ Smoluchowskiego 17, 60-179 Poznań, Poland \\ AND J. BARNAŚ \\ Magnetism Theory Division, Institute of Physics, A. Mickiewicz University \\ Matejki 48/49, 60-769 Poznań, Poland
}

General quantum-mechanical description of electronic transport in thin-film structures, which is based on the Kubo approach, is presented and applied to a single film with ideal surfaces. The cases of a constant chemical potential and a constant particle number are considered and analysed numerically.

PACS numbers: 73.50.-h, 73.61.At

Transport in thin-film structures (single films and multilayers) is of current interest [1-4] due to new interesting phenomena found in such systems. Quasiclassical description based on the Boltzmann transport equation is very often applied to such structures, however, it underestimates the electron scattering by surfaces and/or interfaces. In this paper we formulate a general quantum mechanical description of electronic transport in thin-film structures. The approach is based on the single electron transport theory which was applied to many problems like weak localization, universal conductance fluctuations etc. [5]. The main contribution to the global in-plane conductivity is given, for each spin direction, by the formula

$$
\sigma=\frac{1}{l} \int \mathrm{d} z \sigma(z),
$$

where the axis $z$ is assumed to be normal to the structure and $l$ is the total thickness of the structure. The local conductivity $\sigma(z)$ is given by the expression

$$
\begin{aligned}
\sigma(z) & =-\frac{1}{4}\left(\frac{e}{m}\right)^{2} \frac{\hbar}{(2 \pi)^{2}} \int \mathrm{d} q \int \mathrm{d} z^{\prime} q^{3} \\
& \times\left(G_{q}^{\mathbf{R}}\left(z, z^{\prime}\right)-G_{q}^{\mathbf{A}}\left(z, z^{\prime}\right)\right)\left(G_{q}^{\mathbf{R}}\left(z^{\prime}, z\right)-G_{q}^{\mathbf{A}}\left(z^{\prime}, z\right)\right)
\end{aligned}
$$

with $G_{q}^{\mathrm{R}}\left(z, z^{\prime}\right)$ and $G_{q}^{\mathrm{A}}\left(z, z^{\prime}\right)$ being the retarded and advanced Green's functions taken at the Fermi energy $E_{F}$ and averaged over impurity distribution. They are 
written here in the mixed $(q, z)$ representation. The impurity averaged Green's function $G_{q}^{\mathrm{R}}\left(z, z^{\prime}\right)$ fulfils the following differential equation:

$$
\frac{\hbar^{2}}{2 m} \frac{\mathrm{d}^{2}}{\mathrm{~d} z^{2}}+\left(E_{\mathrm{F}}-\frac{\hbar^{2} q^{2}}{2 m}+\mathrm{i} \frac{\hbar}{2 \tau(z)}\right) G_{q}^{\mathrm{R}}\left(z, z^{\prime}\right)=\hbar \delta\left(z-z^{\prime}\right),
$$

where the relaxation time is determined by the imaginary part of the corresponding self-energy $\Sigma(z)$

$$
\frac{1}{2 \tau(z)}=-\operatorname{Im} \Sigma(z)
$$

and is spin- and $z$-dependent, in general.

Consider a thin film of thickness $l$ with the ideal surfaces placed at $z=0$ and $z=l$. To calculate $G_{q}^{\mathrm{R}}\left(z, z^{\prime}\right)$, we use Eq. (3) and the hard wall boundary conditions

$$
G_{q}^{\mathrm{R}}\left(z, z^{\prime}\right)=0 \quad \text { at } \quad z=0, l
$$

which take into account the level quantization due to the external confining potential.

For a single film one finds

$$
G_{q}^{\mathrm{R}}\left(z, z^{\prime}\right)=-\frac{2 m}{\hbar} \frac{1}{k \sin (k l)} \begin{cases}\sin (k z) \sin \left[k\left(1-z^{\prime}\right)\right], & z<z^{\prime}, \\ \sin \left(k z^{\prime}\right) \sin [k(1-z)], & z>z^{\prime},\end{cases}
$$

where $k$ is defined as

$$
k=\left(\frac{2 m}{\hbar^{2}} E_{\mathrm{F}}-q^{2}+\mathrm{i} \frac{m}{\hbar \tau}\right)^{1 / 2}
$$

and is complex in general, $k=k^{\prime}+\mathrm{i} k^{\prime \prime}$. We assumed that the relaxation time is uniform across the film. Green's function $G_{q}^{\mathrm{A}}\left(z, z^{\prime}\right)$ can be found from the relation

$$
G_{q}^{\mathrm{A}}\left(z, z^{\prime}\right)=G_{q}^{\mathrm{R}}\left(z^{\prime}, z\right)^{*} .
$$

Taking into account Eq. (1) and Eq. (2) one finds for $\sigma$ the following expression:

$$
\sigma=\sigma_{1}+\sigma_{2}=\frac{1}{2 l}\left(\frac{e}{m}\right)^{2} \frac{\hbar}{(2 \pi)^{2}} \int \mathrm{d} q q^{3}\left(\sigma_{q}^{(1)}+\sigma_{q}^{(2)}\right)
$$

where

$$
\begin{aligned}
\sigma_{1 q} & =\int_{0}^{l} \mathrm{~d} z \int_{0}^{l} \mathrm{~d} z^{\prime} G_{q}^{\mathrm{R}}\left(z, z^{\prime}\right) G_{q}^{\mathrm{A}}\left(z^{\prime}, z\right), \\
\sigma_{2 q} & =\operatorname{Re}\left[\int_{0}^{l} \mathrm{~d} z \int_{0}^{l} \mathrm{~d} z^{\prime} G_{q}^{\mathrm{R}}\left(z, z^{\prime}\right) G_{q}^{\mathrm{R}}\left(z^{\prime}, z\right)\right] .
\end{aligned}
$$

Taking into account the explicit forms of Green's functions one finds

$$
\begin{aligned}
\sigma_{1 q} & =\frac{8 m l^{4}}{\hbar^{2}}\left\{\frac{\sinh x^{\prime \prime}}{x^{\prime \prime}}+\frac{\sin x^{\prime}}{x^{\prime}}-4 \frac{\cosh x^{\prime \prime}-\cos x^{\prime}}{x^{\prime 2}+x^{\prime \prime}}\right\} \\
& \times\left[\left(x^{\prime 2}+x^{\prime 2}\right)\left(\cosh x^{\prime \prime}-\cosh x^{\prime}\right)\right]^{-1} \\
\sigma_{2 q} & =\frac{-8 m l^{4}}{\hbar^{2}}\left[\frac{\left(x^{\prime 2}-x^{\prime \prime}\right)\left[1-\cos x^{\prime} \cosh x^{\prime \prime}\right]-2 x^{\prime} x^{\prime \prime} \sin x^{\prime} \sinh x^{\prime \prime}}{\left(\cosh x^{\prime \prime}-\cos x^{\prime}\right)^{2}\left(x^{\prime 2}+x^{\prime 2}\right)^{2}}\right.
\end{aligned}
$$




$$
\begin{aligned}
& +\frac{\left({x^{\prime}}^{3}-3 x^{\prime} x^{\prime 2}\right) \sin x^{\prime}+\left(x^{\prime 3}-3{x^{\prime}}^{2} x^{\prime \prime}\right) \sinh x^{\prime \prime}}{\left(\cosh x^{\prime \prime}-\cos x^{\prime}\right)\left({x^{\prime}}^{2}+{x^{\prime \prime}}^{2}\right)^{3}} \\
& \left.-4 \frac{\left({x^{\prime}}^{2}+{x^{\prime \prime}}^{2}\right)^{2}-8{x^{\prime}}^{2} x^{\prime 2}}{\left({x^{\prime}}^{2}+{x^{\prime \prime}}^{2}\right)^{4}}\right]
\end{aligned}
$$

where we have defined

$$
\begin{aligned}
& x^{\prime}=2 k^{\prime} l, \\
& x^{\prime \prime}=2 k^{\prime \prime} l .
\end{aligned}
$$

Integration over $q$ in Eq. (8) has been performed numerically. In Fig. 1 we see the

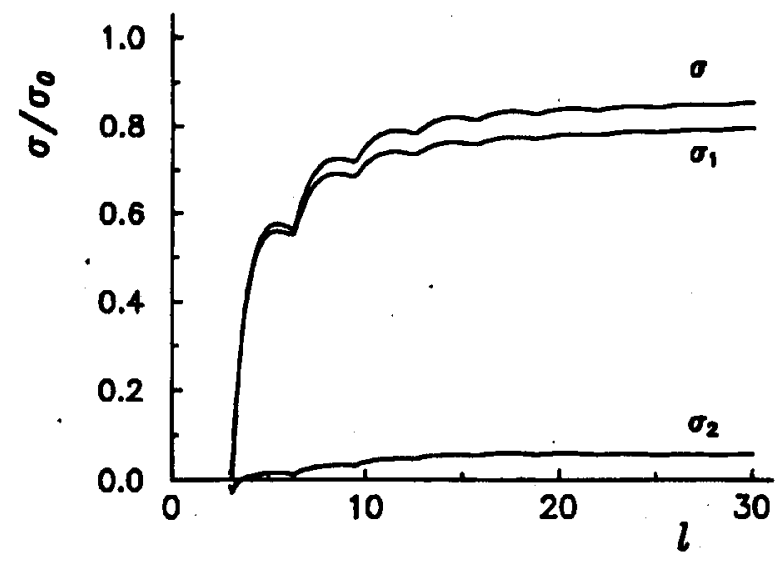

Fig. 1. Relative conductivity $\sigma / \sigma_{0}$ vs. film thickness $l$ (measured in arbitrary units) for a constant Fermi energy $E_{\mathrm{F}}=1$ (measured in units with $\hbar^{2} / 2 m=1$ ) and mean free path $l_{s}=100$. The contributions $\sigma_{1} / \sigma_{0}$ and $\sigma_{2} / \sigma_{0}$ are also presented.

contributions $\sigma_{1}$ and $\sigma_{2}$ as well as the total conductivity $\sigma$, which are normalized to the Drude conductivity $\sigma_{0}=n e^{2} \tau / m$ and shown as a function of the film thickness. The curves have been calculated for a constant chemical potential. The following features are noteworthy. (1) The conductivity shows a fine structure with the increasing film thickness and the distance between two consecutive dips is equal to half of the Fermi wavelength. (2) The contribution $\sigma_{2}$ does not vanish and is of the order of $0.1 \sigma_{1}$ for the parameters assumed there. The contribution $\sigma_{2}$ is usually neglected but it is clear that it does not vanish and may be quite considerable.

The fine structure of the conductivity shown in Fig. 1 comes from the quantization of the perpendicular motion and from the fact that the chemical potential is kept constant. When the total particle number in the system is conserved, the Fermi energy behaves as shown in Fig. 2. The aonductivity is now independent of the film thickness. Such behaviour takes place when there is no surface scattering. If surface roughness is important then the quantum size effects are evident in the 


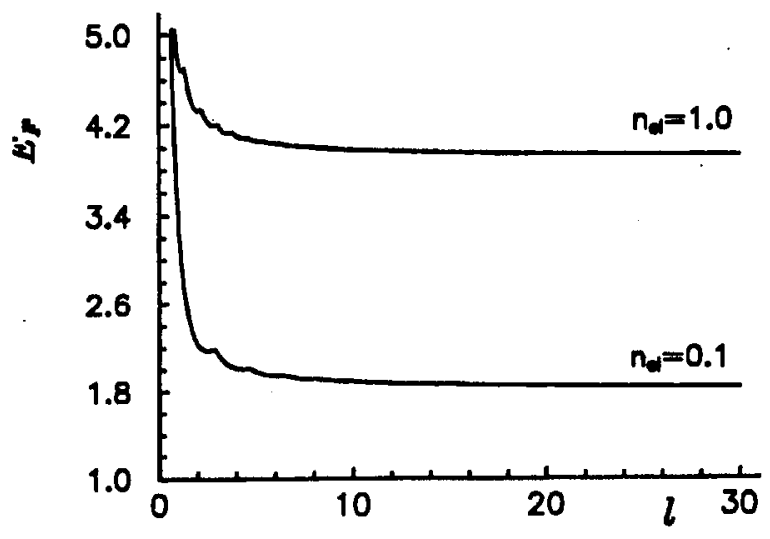

Fig. 2. Fermi energy (measured in units with $\hbar^{2} / 2 m=1$ ) vs. film thickness for given electron concentrations.

conductivity which decreases with decreasing film thickness. The surface roughness can be simulated within the formalism described above by a surface layer on both sides of the film with a sufficiently higher impurity concentration, i.e. with $\tau$ larger than inside the film.

\section{References}

[1] N. Trivedi, N.W. Ashcroft, Phys. Rev. B 38, 12298 (1988).

[2] Z. Tesanovic, J. Phys. C, Solid State Phys. 20, L829 (1987).

[3] G. Fishman, D. Calecki, Phys. Rev. Lett. 62, 1302 (1989).

[4] H.E. Camblong, P.M. Levy, Phys. Rev. Lett. 69, 2835 (1992).

[5] J. Ramer, Rev. Mod. Phys. 63, 781 (1991). 